\title{
Estimating virtual neck circumference for preoperative STOP-BANG assessment: a retrospective cohort study
}

\author{
Calvin Gruss, MS $\cdot$ Maxim A. Terekhov, MS $\cdot$ Jesse M. Ehrenfeld, MD, MPH $\cdot$ Russell J. Kunic, FNP-BC • \\ Jonathan P. Wanderer, MD, MPH
}

Received: 21 January 2015/Revised: 7 September 2015/Accepted: 14 September 2015/Published online: 18 September 2015

(c) Canadian Anesthesiologists' Society 2015

\section{To the Editor,}

Obstructive sleep apnea (OSA) is associated with increased perioperative morbidity and mortality, ${ }^{1}$ and moderate-severe OSA remains undiagnosed in up to $82 \%$ of males and $92 \%$ of females. ${ }^{2}$ Risk for OSA is commonly assessed using the STOP-BANG tool, which requires a measurement of neck circumference. ${ }^{3}$ There is currently no established method to assess patient neck circumference virtually (i.e., via electronic communication). With increasing numbers of patients having access to techn ology, telemedicine may be able to replace conventional on-site preoperative clinic assessments with convenient and cost-effective virtual patient assessments. Therefore, we investigated using estimates of neck circumference, derived virtually from previously collected photographs, to replace the traditional preoperative clinic measurements.

With institutional review board approval, we completed a retrospective cohort study at the Vanderbilt Preoperative Evaluation Clinic from March 1, 2013 to March 1, 2014. We selected all patients having two preoperative asse

C. Gruss, MS ( $)$

Department of Anesthesiology, Vanderbilt University School of Medicine, Nashville, TN, USA

e-mail: calvin.l.gruss@vanderbilt.edu

M. A. Terekhov, MS · J. M. Ehrenfeld, MD, MPH ·

J. P. Wanderer, MD, MPH

Department of Biomedical Informatics, Vanderbilt University

School of Medicine, Nashville, TN, USA

J. M. Ehrenfeld, MD, MPH · J. P. Wanderer, MD, MPH Department of Urology, Vanderbilt University School of Medicine, Nashville, TN, USA

R. J. Kunic, FNP-BC

Vanderbilt University School of Medicine, Nashville, TN, USA ssments within the study period (Cohort-1). Though duplicating assessments is not standard practice in the clinic, some patients receive multiple preoperative asses sments when they undergo multiple unrelated procedures or repeat procedures or experience extended delays between assessment and surgery. We excluded patients with more than a $10 \%$ change in weight between visits. At each preoperative visit, a clinic technician used a tape measure to determine the patients' neck circumference measurement. We then compared the two neck circum ference measurements.

We separately selected a patient population (Cohort-2) of 110 individuals with both a preoperative facial photograph (which we routinely obtain) and an in-clinic neck circumference measurement. Each patient's photo graph was taken with an iPad and wirelessly transmitted into their medical record. We excluded patients from the study if their neck view was obstructed in the photograph. Using this preoperative image, we estimated a virtual neck circumference and compared our calculation with the neck circumference measurement (taken on-site) for analysis. The photograph included a one-inch square reference image in the background used to scale the photograph according to pixel length (Figure A). We used the following equation to determine an estimate for the neck circumference (which we assumed was circular):

$\frac{\text { Pixel Length }_{\text {Neck }}}{\text { Pixel Length }}$ Reference Image $_{\text {Inch Length }}^{\text {Reference Image }}$
Neck Circumference $=\pi \cdot$ Neck Diameter

We then categorized each measurement and estimate as $\leq 40 \mathrm{~cm}$ or $>40 \mathrm{~cm}$ (i.e., the positive test threshold in the STOP-BANG criteria) and compared the measurements with the estimates in Cohort-2 using a McNemar's test and 
a Bland-Altman plot. Agreement between the measurements was assessed using the concordance correlation coefficient. ${ }^{4}$

The mean (SD) difference between the two neck circumference measurements in the 591 patients in Cohort1 was $-0.2(2.2) \mathrm{cm}$ (Figure B), which is consistent with research completed in pediatric populations ${ }^{5}$ [concordance correlation coefficient, $0.904 ; 95 \%$ confidence interval (CI), 0.888 to 0.917$]$.

The mean (SD) BMI of Cohort-2 was 29.5 (6.6) $\mathrm{kg} \cdot \mathrm{m}^{-2}$. The mean (SD) difference between the estimate and the measurement of the 82 images analyzed from the 110 patients in Cohort-2 was -0.7 (2.6) $\mathrm{cm}$ (concordance correlation coefficient, $0.838 ; 95 \% \mathrm{CI}, 0.759$ to 0.892 ) (Figure C). As for the $\leq 40 \mathrm{~cm}$ or $>40 \mathrm{~cm}$ categorization in Cohort-2, there was no significant difference between the

A
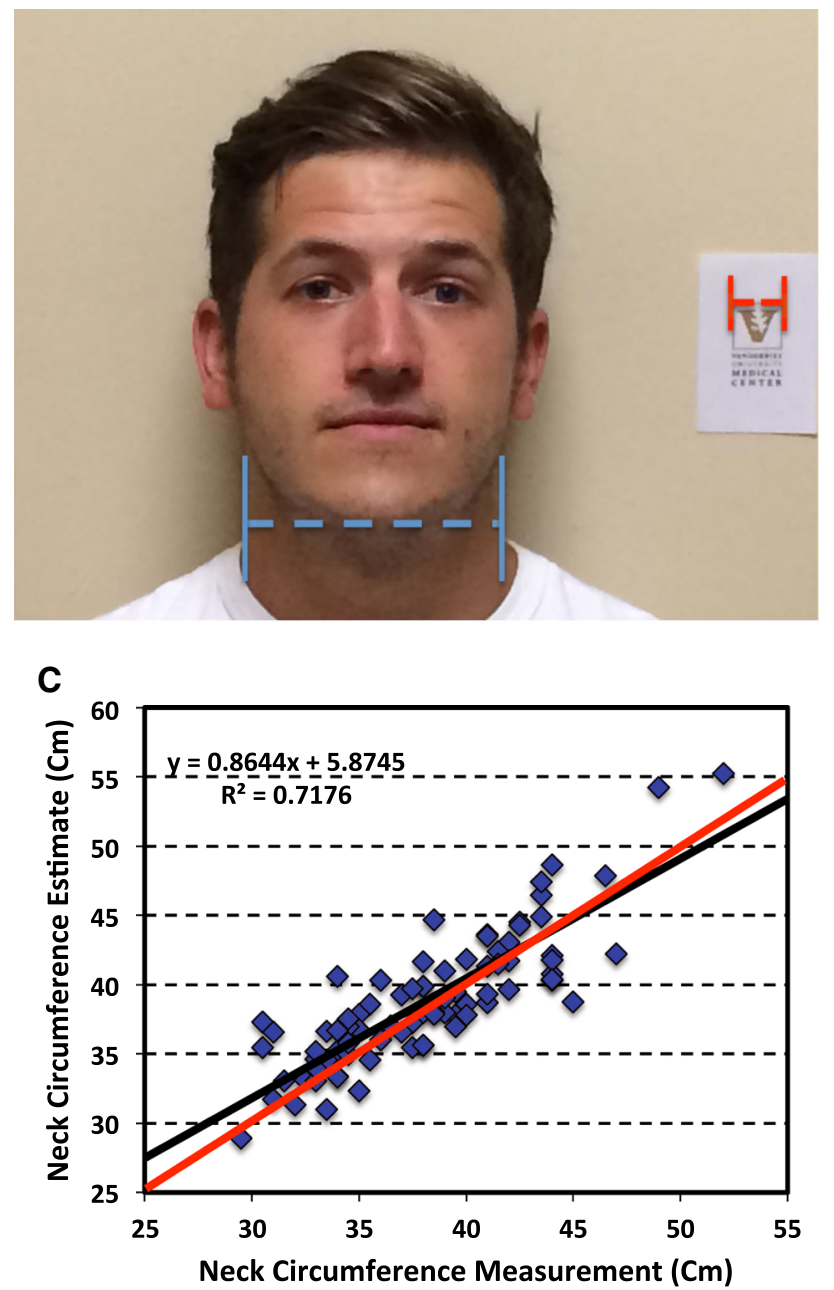

Figure (A) Patient photograph (with permission) for virtual neck circumference estimation. Head positioning relative to the camera is important to make an accurate estimation of neck diameter (dashed blue line) relative to the reference image (dashed red line). (B) Precision of repeated neck measurements taken with a tape measure for patients undergoing an on-site preoperative evaluation shown with measurements and the virtual estimates $(P=0.5271)$. The Bland-Altman plot was used to calculate a prediction interval for the difference between the measurement and the estimate (mean difference, $-0.7 \mathrm{~cm}$; 95\% CI, -6.2 to 6.8) (Figure D).

The difference between the two tape measurements in Cohort-1 appears similar to the difference between the estimate and a measurement in Cohort-2. Using an estimate to assess patients' neck circumference preoperatively looks to be a reliable alternative to repeated neck measurements. Ideally, this estimate would be integrated into a compre hensive virtual evaluation, and on-site preoperative evaluation clinics would need to see fewer patients prior to the day of surgery. This procedure would help streamline the perioperative process and save the patient and institution valuable time and resources.

B
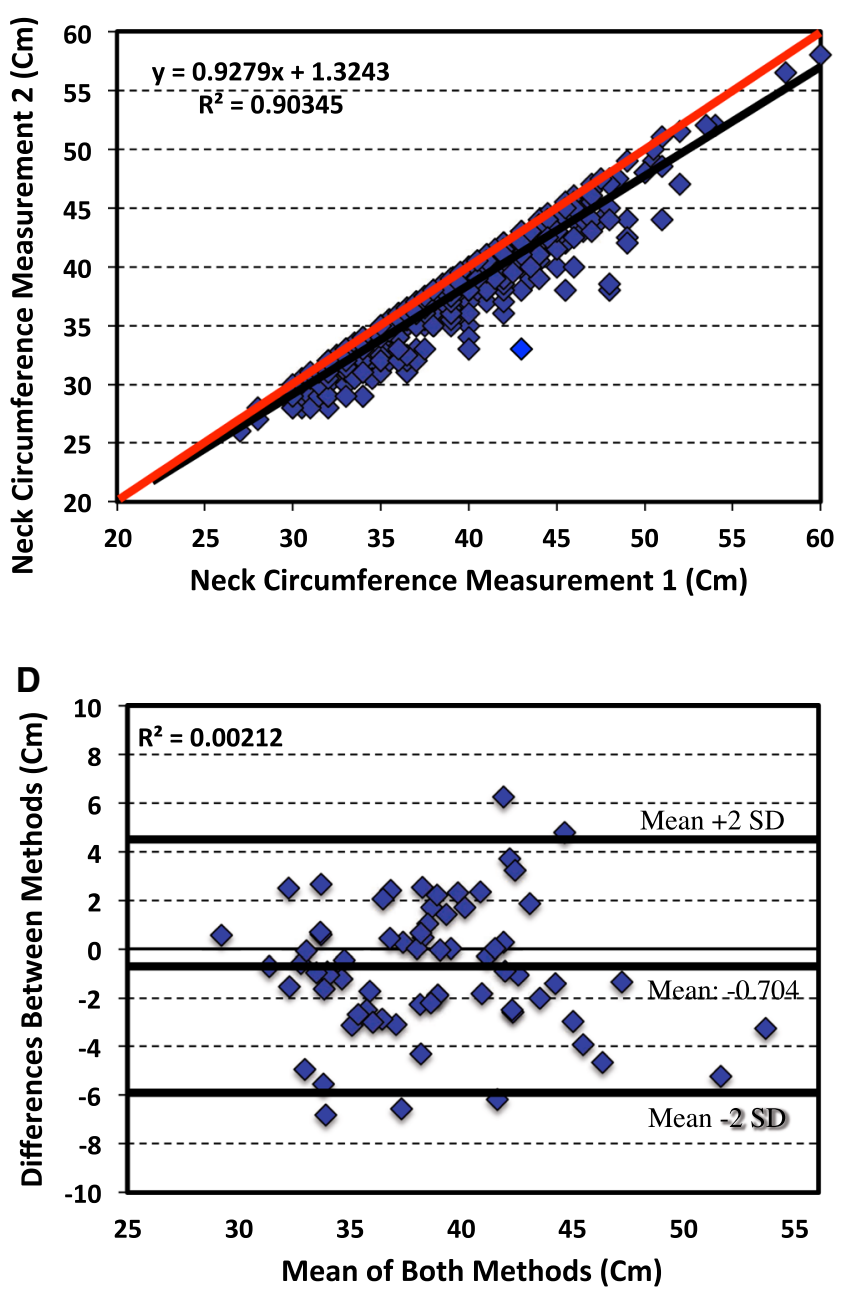

$45^{\circ}$ line (red line) for comparison $(n=591)$. (C) Precision of a virtual neck circumference estimation compared with a neck circumference measurement taken with a tape measure for patients shown with $45^{\circ}$ line (red line) for comparison $(n=82)$. (D) Bland-Altman plot comparing the virtual neck circumference estimation with a neck circumference measurement taken with a tape measure $(n=82)$ 
Conflicts of interest None declared.

Funding Dr. Wanderer is supported by the Foundation for Anesthesia Education and Research (FAER) and the Anesthesia Quality Institute (AQI), Mentored Research Training Grant in Health Services Research (MRTG-HSR). This study was supported in part by the Foundation for Anesthesia Education and Research as well as by departmental funding.

\section{References}

1. Boushra NN. Anaesthetic management of patients with sleep apnoea syndrome. Can J Anaesth 1996; 43: 599-616.
2. Young T, Evans L, Finn L, Palta M. Estimation of the clinically diagnosed proportion of sleep apnea syndrome in middle-aged men and women. Sleep 1997; 20: 705-6.

3. Chung F, Yegneswaran B, Liao $P$, et al. STOP questionnaire: a tool to screen patients for obstructive sleep apnea. Anesthesiology 2008; 108: 812-21.

4. Lin LI. A concordance correlation coefficient to evaluate reproducibility. Biometrics 1989; 45: 255-68.

5. LaBerge RC, Vaccani JP, Gow RM, Gaboury I, Hoey L, Katz SL. Inter- and intra-rater reliability of neck circumference measurements in children. Pediatr Pulmonol 2009; 44: 64-9. 